\title{
Optimal design of 2-Bit Optical Magnitude Comparator using electro-optic effect inside Mach-Zehnder interferometers
}

\author{
Kuldeep Choudhary, Santosh Kumar \\ Photonics Lab \\ Department of Electronics \\ and Communication Engineering \\ DIT University \\ Dehradun, Uttarakhand-248009, India
}

\begin{abstract}
In this paper, 2-bit magnitude comparator using electro-optic effect inside lithium-niobate based Mach-Zehnder interferometers (MZI) is proposed. The design is based on six MZIs only, hence proposed device is optimized in comparison of previous design. The mathematical description of device is well described. The results are verified using MATLAB and Beam propagation method simulation.
\end{abstract}

Index Terms-Lithium-niobate, Mach-Zehnder interferometers, Beam propagation method.

\section{INTRODUCTION}

In recent years, interferometric based optical switches have been explored in switching area largely. As light propagates through the Mach-Zehnder interferometer (MZI) means switching speed is high. MZI rooted on electro-optic effect in LiNbO3 appears to be an optimistic solution [1-3], because of its quality attributes of compact size, thermal stability [1], re-configurability [2], integration potential [1], low latency [3] and low power consumption.

In this paper, a new approach is used to realize an optimal design of 2-bit magnitude comparator that reduces the complexity of the previous design [14] because in pervious design there were 12 MZI's required but in our design only six MZI's are used.

\section{Proposed design of 2-Bit optical magnitude comparator}

Fig. 1 is showing the structure of 2-bit optical magnitude comparator, implemented by using only six MZIS. A single optical continuous source is applied to the whole design which is propagated from first input port of first MZI mentioned as MZI1 to the last MZI i.e MZI6 following the various combination depending upon the different combinations of the control signal provided to the electrodes in the MZI. On the basis of 2-bit control signal $A$ and $B$, there are three different outputs and that are; $A>B$ which is obtained at output port 3 feeding directly with MZI6, second output of the proposed device is $A=B$ which is available with MZI5 at its first output port and represented by port 1, third output of the proposed device is $A<B$ which can be extracted by using the outputs of MZI4 and MZI6 and finally at output port 2 .

\section{A. Mathematical expressions of the different output ports}

Normalized power at different output ports can be calculated and used to verify the Opti-BPM results using MATLAB.

Using figure 1, the normalized power for output port 1 $(\mathrm{A}=\mathrm{B})$ can be calculated as:

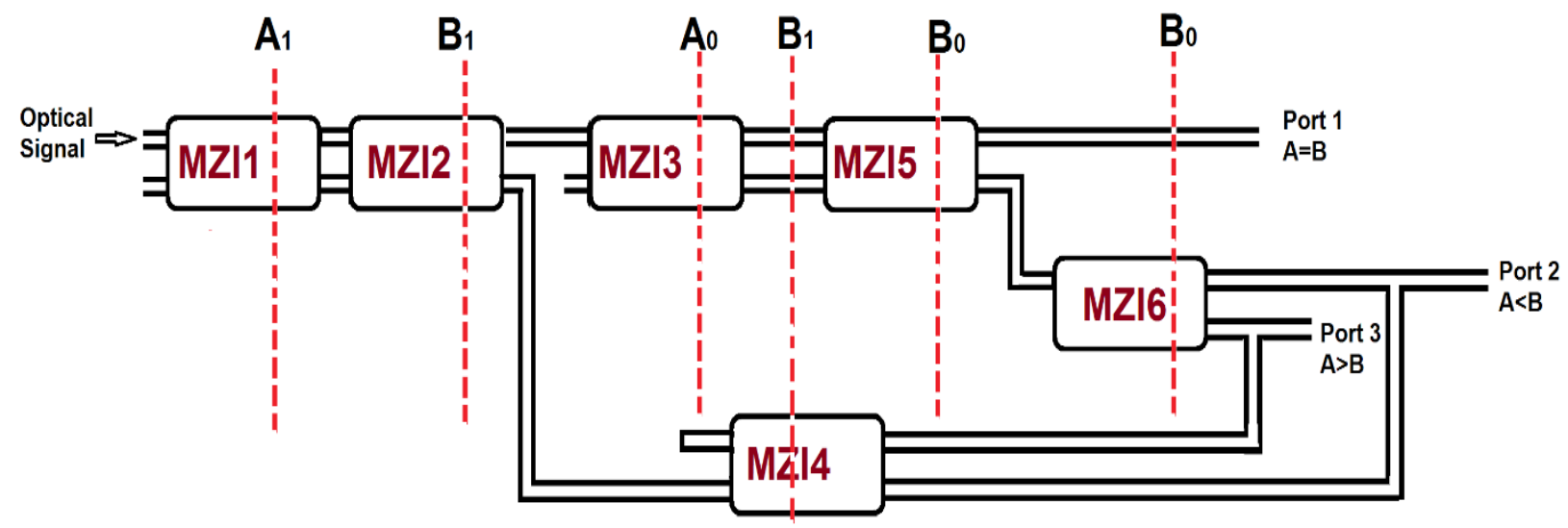

Fig. 1. Design of Optimal 2-bit optical magnitude comparator using the MZIs. 


\section{Output Port $1=$}

$$
\begin{array}{r}
\left\{\sin ^{2}\left(\frac{\Delta \varphi_{M Z I 1}}{2}\right) \sin ^{2}\left(\frac{\Delta \varphi_{M Z I 2}}{2}\right)+\cos ^{2}\left(\frac{\Delta \varphi_{M Z I 1}}{2}\right) \cos ^{2}\left(\frac{\Delta \varphi_{M Z I 2}}{2}\right)\right\} \\
\left\{\cos ^{2}\left(\frac{\Delta \varphi_{M Z I 3}}{2}\right) \cos ^{2}\left(\frac{\Delta \varphi_{M Z I 5}}{2}\right)+\sin ^{2}\left(\frac{\Delta \varphi_{M Z I 3}}{2}\right) \sin ^{2}\left(\frac{\Delta \varphi_{M Z I 5}}{2}\right)\right\}
\end{array}
$$

In the continuation, the normalized power of port 2 collectively obtained by MZI4 and MZI6 as shown below:

\section{Output Port 2 =}

$$
\begin{gathered}
\left\{\sin ^{2}\left(\frac{\Delta \varphi_{M Z I 1}}{2}\right) \sin ^{2}\left(\frac{\Delta \varphi_{M Z I 2}}{2}\right)+\cos ^{2}\left(\frac{\Delta \varphi_{M Z I 1}}{2}\right) \cos ^{2}\left(\frac{\Delta \varphi_{M Z I 2}}{2}\right)\right\} \\
\left\{\sin ^{2}\left(\frac{\Delta \varphi_{M Z I 3}}{2}\right) \sin ^{2}\left(\frac{\Delta \varphi_{M Z I 5}}{2}\right) \sin ^{2}\left(\frac{\Delta \varphi_{M Z I 6}}{2}\right)\right\} \\
+\left\{\cos ^{2}\left(\frac{\Delta \varphi_{M Z I 1}}{2}\right) \sin ^{2}\left(\frac{\Delta \varphi_{M Z I 2}}{2}\right) \sin ^{2}\left(\frac{\Delta \varphi_{M Z I 4}}{2}\right)\right\}
\end{gathered}
$$

Similarly,

\section{Output Port 3 OUT3=}

$$
\begin{gathered}
\left\{\sin ^{2}\left(\frac{\Delta \varphi_{M Z I 1}}{2}\right) \sin ^{2}\left(\frac{\Delta \varphi_{M Z I 2}}{2}\right)+\cos ^{2}\left(\frac{\Delta \varphi_{M Z I 1}}{2}\right) \cos ^{2}\left(\frac{\Delta \varphi_{M Z I 2}}{2}\right)\right\} \\
\quad\left\{\sin ^{2}\left(\frac{\Delta \varphi_{M Z I 3}}{2}\right) \cos ^{2}\left(\frac{\Delta \varphi_{M Z I 5}}{2}\right) \cos ^{2}\left(\frac{\Delta \varphi_{M Z I 6}}{2}\right)\right\}
\end{gathered}
$$

The above three equations are used to obtained the MATLAB results of the proposed device, where two control signals $\mathrm{A}$ and $\mathrm{B}$ are compared and applied at second electrode of the MZIs B1, A1 and B0, A0 are representing the MSB and LSB bits of numbers $B$ and A respectively. As shown in Fig 3 when both the control inputs are at $0 \mathrm{~V}$ (low) then the signal gets at output port $\mathrm{A}=\mathrm{B}$ (high) and all other output port have no signal. When $\mathrm{A} 0$ is increasing from $0 \mathrm{~V}$ to $6.75 \mathrm{~V}$ and

$\mathrm{B} 1=\mathrm{A} 1=\mathrm{B} 0=0 \mathrm{~V}$, then signal intensity at port $(\mathrm{A}=\mathrm{B})$ is decreasing and output at $(\mathrm{A}>\mathrm{B})$ is increasing. Similarly, all other cases can be described from Fig 2 .

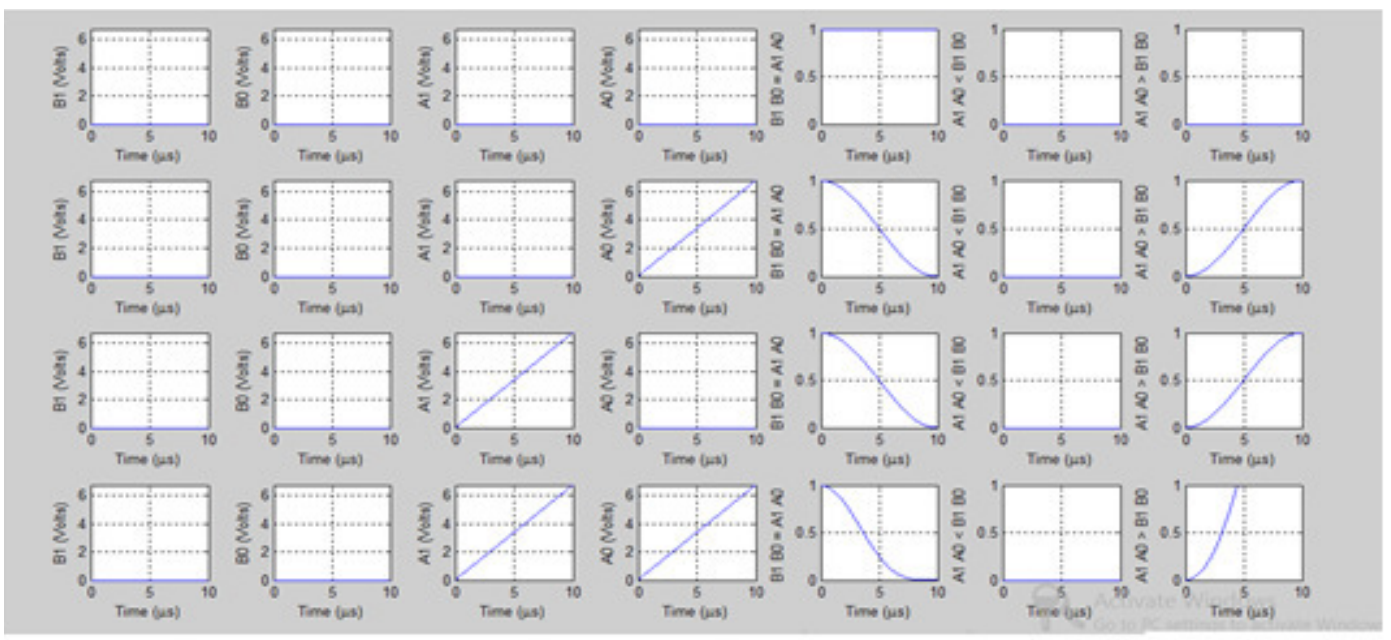

(a)
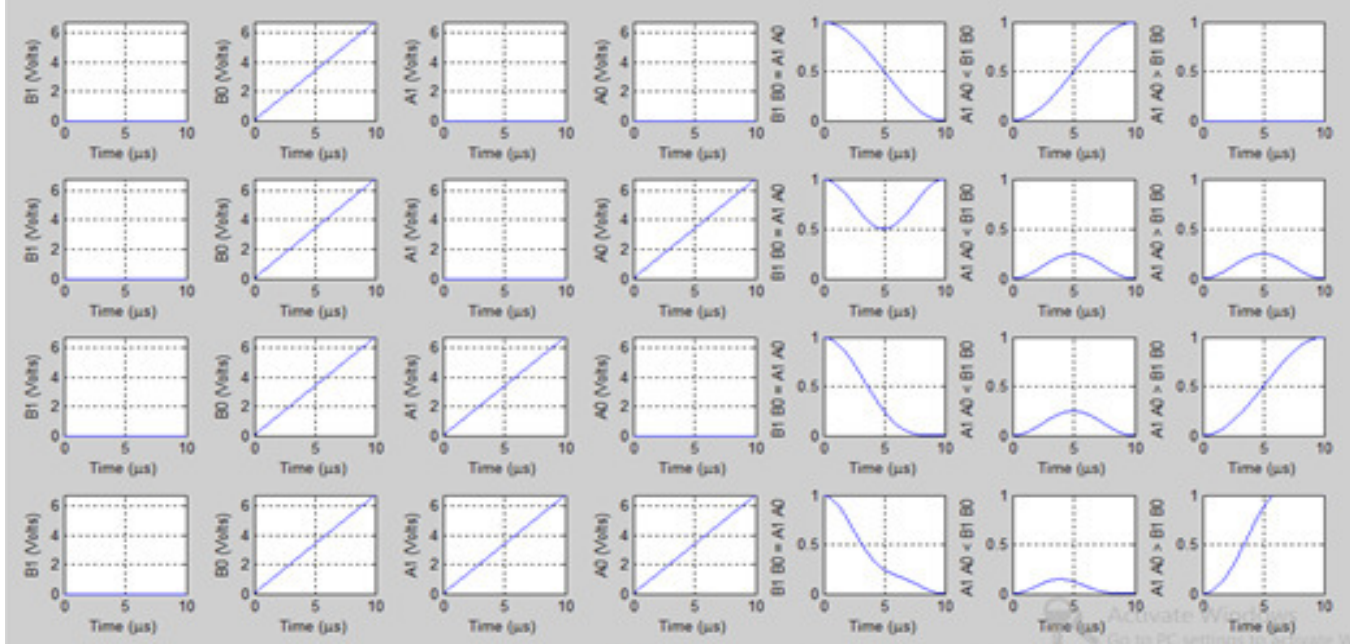

(b) 


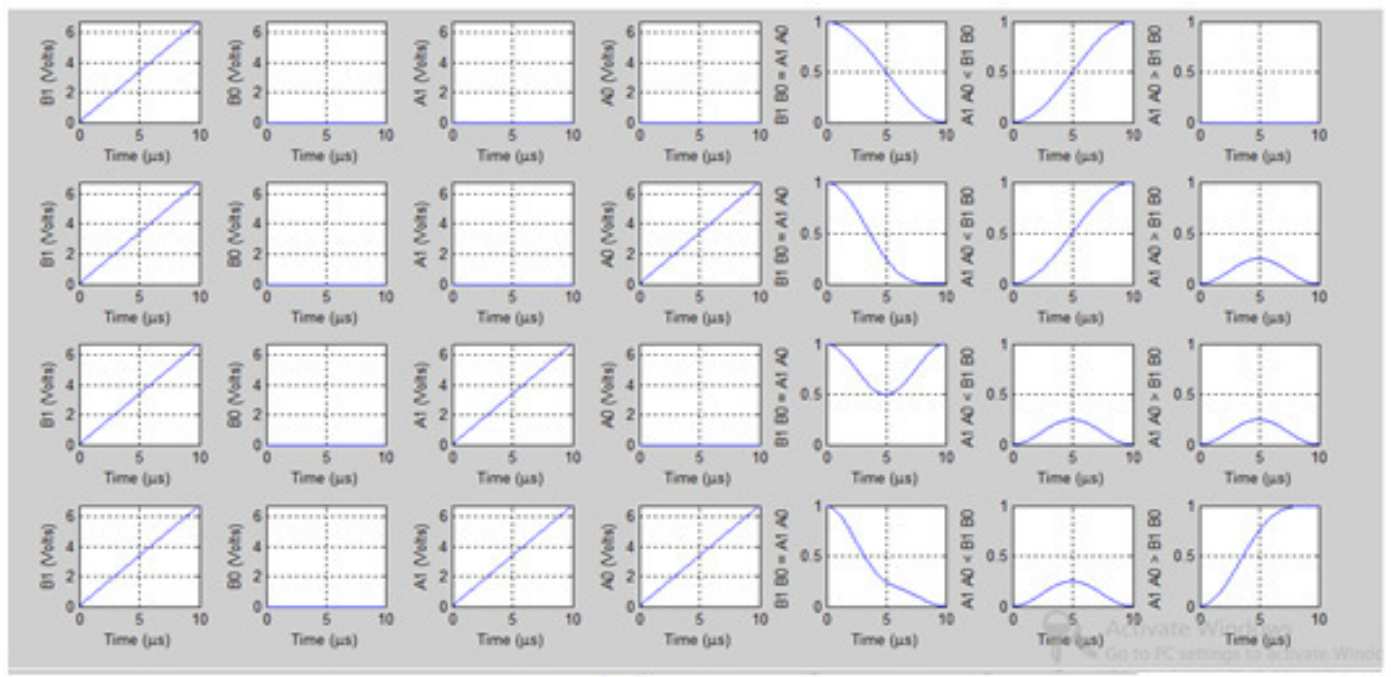

(c)

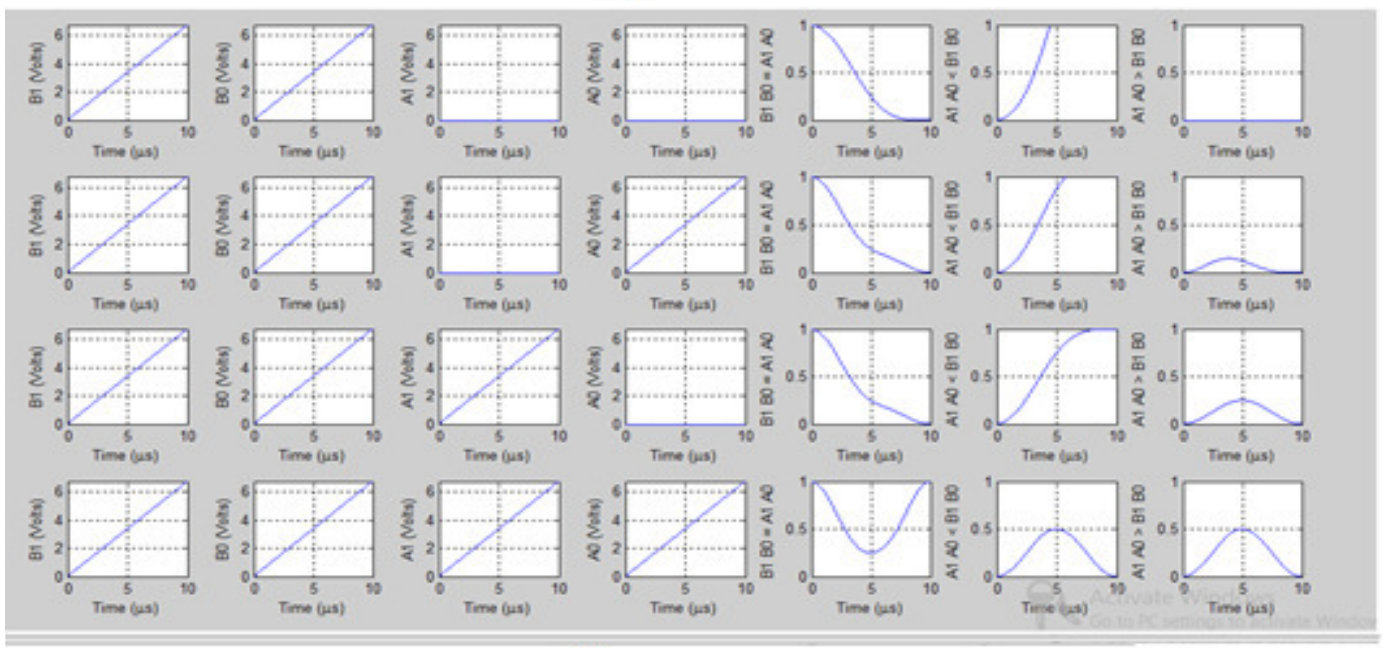

(d)

Fig. 2. MATLAB simulation results of optimal 2-bit magnitude comparator for different combinations of control signals, B is (a) 0 (b) 1 (c) 2 (d) 3 and magnitude of control signal A changes from 0 to 3.

III. Opti-BPM DESIGN OF the Optimal 2-Bit OPtical MAgnitude COMPARATOR

BPM is used to examine the proposed device. Mainly it works on the principle of finite difference Beam propagation method (FD-BPM).

The schematic layout diagram showing in fig 3 is used for comparing two 2-bit numbers A and B. In this proposed design we are using only six MZIs and the final outputs are taken by output port $1(\mathrm{~A}=\mathrm{B})$, port $2(\mathrm{~A}<\mathrm{B})$ and port $3(\mathrm{~A}>\mathrm{B})$. An optical signal is applied to first input port of MZI1 which is propagating till the output obtained. As shown in above fig 3 the first output of port of MZI6 and second output of MZI4 gives the result of $\mathrm{A}<\mathrm{B}$ at output port 2. The first output port of MZI5 gives the result of $A=B$ at output port 1 . Third output of the proposed device at port 3 can be obtained with the combination of MZI6 and MZI4, which results in $\mathrm{A}>\mathrm{B}$ as shown in Fig 3.
Opti-BPM results of optimal 2-bit optical magnitude comparator are shown in below Fig 4 and these results can be verified using the truth table of 2-Bit magnitude comparator listed above in Table 1.

The Opti-BPM results shown in Fig 4 can be explained as:
Case 1: $\quad \mathbf{A 1}=\mathbf{0}$
A0 $=\mathbf{0}$
$\mathbf{B 1}=\mathbf{0}$
B0 $=\mathbf{0}$

A single optical signal from continuous wave optical source is feeding to the first input port of MZI1. For this case the control signal to all the MZIs are LOW and therefore light signal is propagating from MZI1 to MZI5 via MZI2 and MZI3. As the control signal at MZI1 is A1 $=0$, the light signal applied to the first input port of MZI1 is emerged at second output port of MZI1. Similarly Light signal propagates from MZI2 (second input port) to MZI2 (first output port) and then from MZI3 (first input port) emerged at second output port of MZI3 and finally light feeding to second input port of MZI5 emerged at first output port of MZI5 and then 


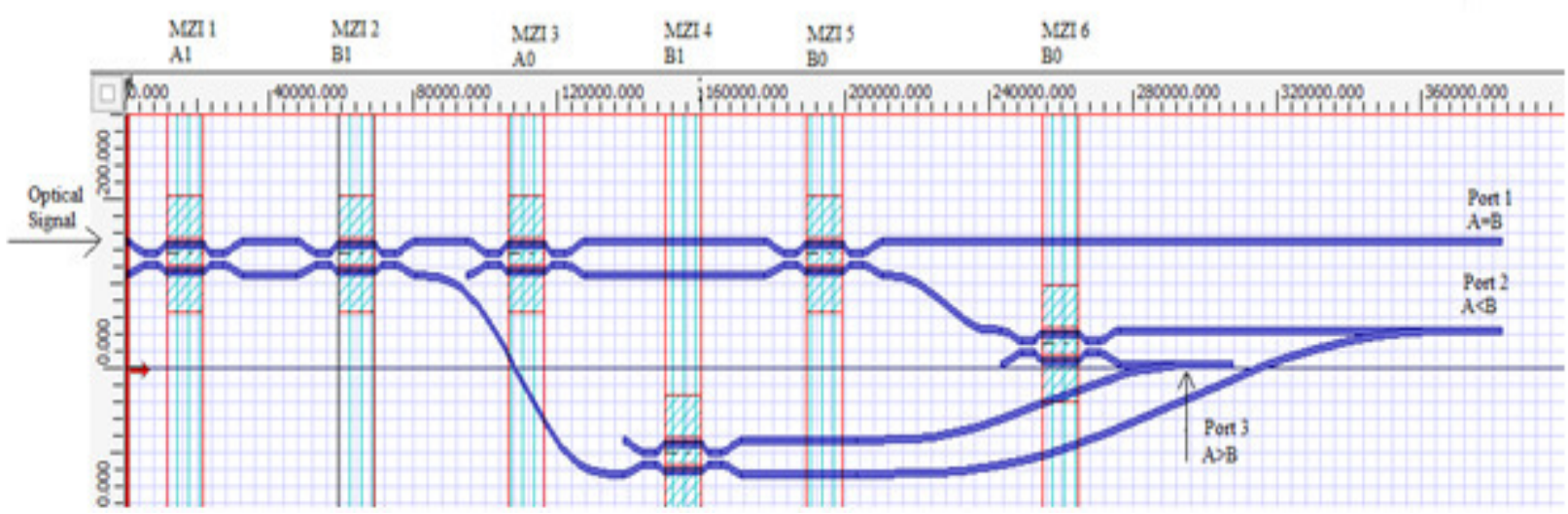

Fig 3. Opti-BPM layout of optimal 2-Bit optical magnitude comparator.

reached to output port 1 as shown in Fig 4(a), verifying the result i.e. $\mathrm{A}=\mathrm{B}$.
Case 2: $\quad A 1=0$
$\mathbf{A 0}=\mathbf{0}$
$\mathbf{B 1}=\mathbf{0}$
B0 $=1$

Continuous wave $(\mathrm{CW})$ optical signal is propagating from MZI1 to MZI6 (first input port) by means of MZI2, MZI3, MZI5. As the control signal, $B 0=1$, the light comes out from first output port of MZI6 and appears on output port 2, executes the logic $\mathrm{A}<\mathrm{B}$.

$$
\text { Case 3: } A 1=0 \quad \text { A0 }=0 \quad \text { B1 }=1 \quad \text { B0 }=0
$$

Impenitent $\mathrm{CW}$ optical signal at MZI1 is arrived to MZI4 by means of MZI2. As the control signal is $\mathrm{B} 1=1$, the light comes out from second output port of MZI4 and executes the $\operatorname{logic} \mathrm{A}<\mathrm{B}$ as shown in Fig 4(a).
Case 4: $\quad A 1=0$
$\mathrm{A0}=\mathbf{0}$
B1 $=1$
B0 $=1$

Inward CW optical signal at MZI1 is arrived to MZI4 by means of MZI2. As the control signal $\mathrm{B} 1=1$, after propagation of light signal it comes out from second output port of MZI4 and meet to the desired result i.e. logic $\mathrm{A}<\mathrm{B}$ as shown in Fig 4(a).

$$
\text { Case 5: } A 1=0 \quad \text { A0 }=1 \quad \text { B1 }=0 \quad \text { B0 }=0
$$

Continuous wave $(\mathrm{CW})$ optical signal is propagating from MZI1 to MZI6 by means of MZI2, MZI3 and MZI5. As $\mathrm{B} 0=0$ applied on the electrode, light comes out from second output port of MZI6 and results in $\mathrm{A}>\mathrm{B}$ as shown in Fig 4(b).

$$
\text { Case 6: } \quad \begin{array}{llll}
A 1=0 & A 0=1 & B 1=0 & B 0=1
\end{array}
$$

Continuous wave $(\mathrm{CW})$ optical signal is propagating from

\begin{tabular}{|c|c|c|c|c|c|c|}
\hline \multicolumn{4}{|c|}{ Control signals } & \multicolumn{3}{|c|}{ Optical output at different ports } \\
\hline \multicolumn{2}{|c|}{$\mathbf{B}$} & \multicolumn{2}{|c|}{$\mathbf{A}$} & Port1 & Port2 & Port3 \\
\hline B1 & $\mathrm{B} 0$ & A1 & $\overline{\mathrm{A} 0}$ & $\mathbf{A}=\mathbf{B}$ & $\mathbf{A}<\mathbf{B}$ & $\mathbf{A}>\mathbf{B}$ \\
\hline 0 & 0 & 0 & 0 & 1 & $\mathbf{0}$ & $\mathbf{0}$ \\
\hline 0 & 0 & 0 & 1 & $\mathbf{0}$ & 0 & 1 \\
\hline 0 & 0 & 1 & 0 & $\mathbf{0}$ & $\mathbf{0}$ & 1 \\
\hline 0 & 0 & 1 & 1 & 0 & 0 & 1 \\
\hline 0 & 1 & 0 & 0 & $\mathbf{0}$ & 1 & $\mathbf{0}$ \\
\hline 0 & 1 & 0 & 1 & 1 & $\mathbf{0}$ & $\mathbf{0}$ \\
\hline 0 & 1 & 1 & 0 & $\mathbf{0}$ & $\mathbf{0}$ & 1 \\
\hline 0 & 1 & 1 & 1 & $\mathbf{0}$ & $\mathbf{0}$ & 1 \\
\hline 1 & 0 & 0 & 0 & 0 & 1 & 0 \\
\hline 1 & 0 & 0 & 1 & $\mathbf{0}$ & 1 & $\mathbf{0}$ \\
\hline 1 & 0 & 1 & 0 & 1 & $\mathbf{0}$ & $\mathbf{0}$ \\
\hline 1 & 0 & 1 & 1 & $\mathbf{0}$ & $\mathbf{0}$ & 1 \\
\hline 1 & 1 & 0 & 0 & $\mathbf{0}$ & 1 & 0 \\
\hline 1 & 1 & 0 & 1 & $\mathbf{0}$ & 1 & 0 \\
\hline 1 & 1 & 1 & 0 & $\mathbf{0}$ & 1 & $\mathbf{0}$ \\
\hline 1 & 1 & 1 & 1 & 1 & $\mathbf{0}$ & $\mathbf{0}$ \\
\hline
\end{tabular}
MZI1 to MZI5 by means of MZI2 and MZI3. As B0=1, the

Table 1: Truth table of 2-bit magnitude comparator 


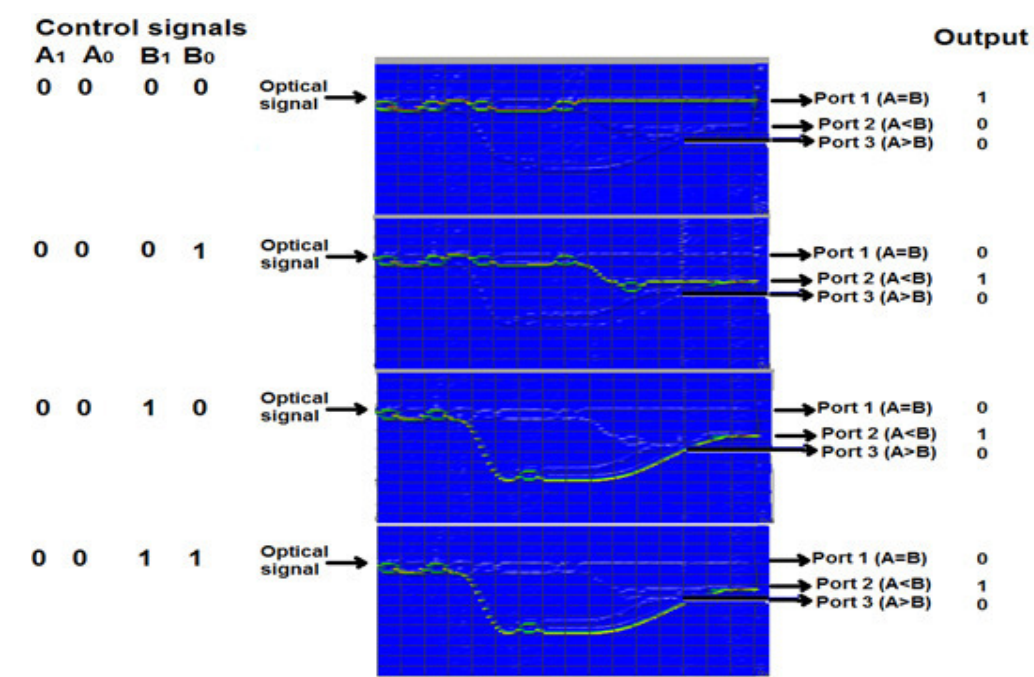

(a)

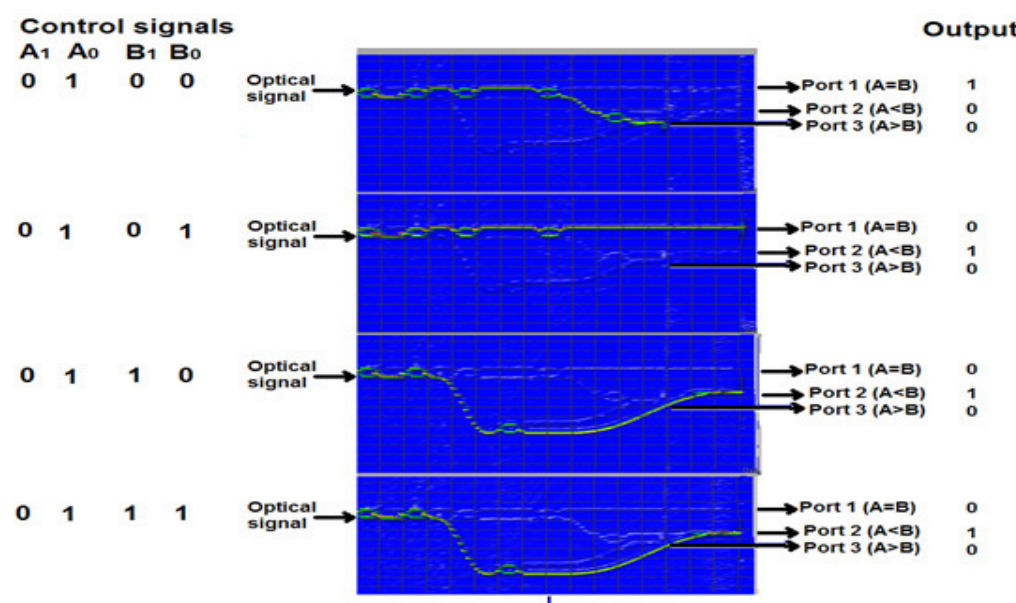

(b)

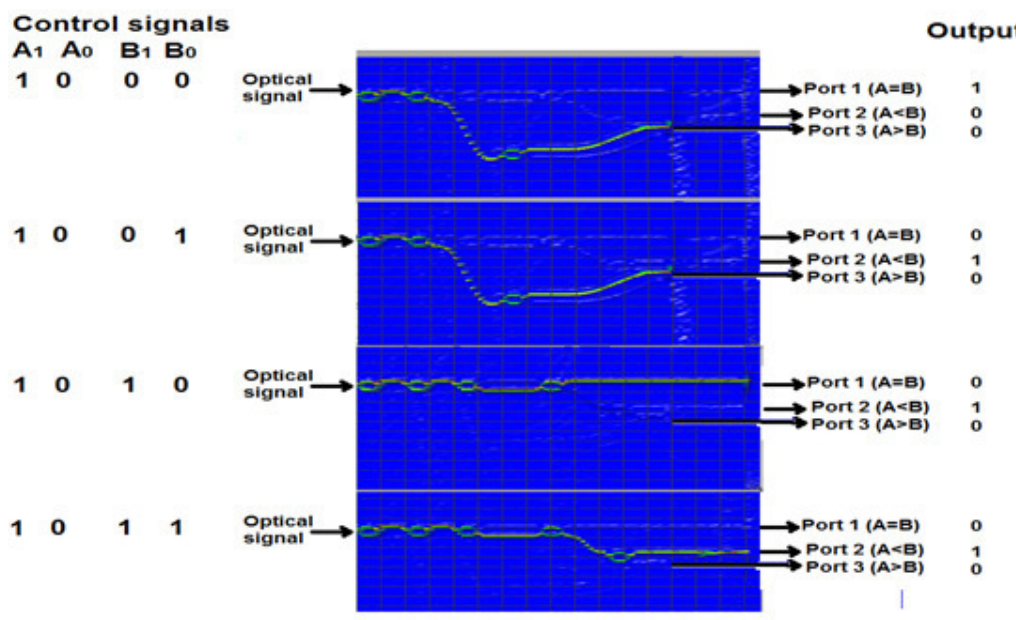

(c)

Fig. 4.Opti-BPM results of optimal 2-bit optical magnitude comparator for different combinations of binary number A is (a) 0 (b) 1 (c) 2 (d) 3 and magnitude of $\mathrm{B}$ changes from 0 to 3 .

light comes from MZI5 (first output port) and results in $\operatorname{logic} \mathrm{A}=\mathrm{B}$ as shown in Fig 4(b).

Case 7: $\quad A 1=0 \quad$ A0 $=1 \quad$ B1 $=1 \quad$ B0 $=0$
Applied CW optical signal at MZI1 is arrived to MZI4 (second input port) by means of MZI2. As the control signal is $\mathrm{B} 1=1$, the light comes out from MZI4 (second output port) and results in logic $A<B$ at port 2 as shown in Fig 4(b). 


\section{Case 8: $\quad A 1=0 \quad A 0=1 \quad B 1=1 \quad B 0=1$}

Continuous wave $(\mathrm{CW})$ optical signal is propagating from MZI1 to MZI4 by means of MZI2. As the control signal is $\mathrm{B} 1=1$, the light comes out from MZI4 (second output port) and executes the logic $\mathrm{A}<\mathrm{B}$ at port 2 as shown in Fig 4(b).

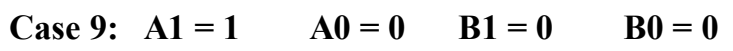

Applied CW optical signal at MZI1 is arrived to MZI4 (second input port) by means of MZI2. As the control signal is $\mathrm{B} 1=0$, the light comes out from MZI4 (first output port) and reached on output port 3 which results in logic $A>B$ as shown in Fig 4(c).

\section{Case 10: $A 1=1 \quad$ A0 $=0 \quad$ B1 $=0 \quad$ B0 $=1$}

Continuous wave $(\mathrm{CW})$ optical signal is propagating from MZI1 to MZI4 by means of MZI2. As the control signal is $\mathrm{B} 1=0$ and $\mathrm{B} 0=1$, the light comes out from MZI4 (first output port) and reached on output port 3 which results in logic $\mathrm{A}>\mathrm{B}$ as shown in Fig 4(c).

\section{Case 11: $A 1=1 \quad$ A0 $=0 \quad$ B1 $=1 \quad$ B0 $=0$}

Applied CW optical signal at MZI1 is arrived to MZI5 (second input port) by means of MZI2 and MZI3. As the control signal at MZI5 is $\mathrm{B} 0=0$, the light comes out from MZI5 (first output port) and reached on output port 1 which results in logic $A=B$ as shown in Fig 4(c).

\section{Case 12: $\quad A 1=1 \quad$ A0 $=0 \quad$ B1 $=1 \quad$ B0 $=1$}

Continuous wave $(\mathrm{CW})$ optical signal is propagating from MZI1 to MZI6 by means of MZI2, MZI3 and MZI5. As the control signal at MZI6 is $\mathrm{B} 0=1$ and $\mathrm{B} 1=1$, the light comes out from MZI6 (first output port) and reached on output port 2 which results in logic $A>B$ as shown in Fig 4(c).

$$
\text { Case 13: } A 1=1 \quad \text { A0 }=1 \quad \text { B1 }=0 \quad \text { B0 }=0
$$

Applied CW optical signal at MZI1 is arrived to MZI4 (second input port) by means of MZI2. As B1=0, the light comes out from MZI4 (first output port) and results in $\mathrm{A}>\mathrm{B}$ as shown in Fig 4(d).

\section{Case 14: $A 1=1 \quad$ A0 $=1 \quad$ B1 $=0 \quad$ B0 $=1$}

Continuous wave $(\mathrm{CW})$ optical signal is propagating from MZI1 to MZI4 by means of MZI2. As the control signal at MZI4 B1=0, the light comes out from MZI4 (first output port) and reached on output port 3 which results in logic $\mathrm{A}>\mathrm{B}$ as shown in Fig 4(d).

\section{Case 15: $A 1=1 \quad$ A0 $=1 \quad$ B1 $=1 \quad$ B0 $=0$}

Applied CW optical signal at MZI1 is arrived to MZI6 (second input port) by means of MZI2, MZI3 and MZI5. As the control signal at MZI6 is $\mathrm{B} 0=0$ and $\mathrm{B} 1=1$, the light comes out from MZI6 (second output port) and reached on output port 2 which results in logic $A>B$ as shown in Fig 4(d).

\section{Case 16: $\quad A 1=1 \quad A 0=1 \quad$ B1 $=1 \quad$ B0 $=1$}

Continuous wave $(\mathrm{CW})$ optical signal is propagating from MZI1 to MZI5 by means of MZI2 and MZI3. As the control signal at MZI5 is $\mathrm{B} 0=1$, the light comes out from MZI5 (first output port) and reached on output port 1 which results in logic $A>B$ as shown in Fig 4(d).

\section{CONCLUSION}

As Electro-optic based Mach-Zehnder Interferometers are become the back bone of optical devices for high speed communication, our proposed device fulfill the requirement of high speed communication in terms of low complexity. Optimization in optical devices helps in fast processing of optical data in the optical devices.

\section{REFERENCES}

[1] S. K. Garai, Method of all-optical frequency encoded decimal to binary and binary coded decimal, binary to gray, and gray to binary data conversion using semiconductor optical amplifiers, Appl. Opt. 50 (2011) 3795-3807.

[2] J. Bhattacharya, A. Gupta, A. Singh, A high performance binary to BCD converter for decimal multiplication, International Symposium on VLSI-DAT IEEE (2010) 315-318.

[3] Y. J. Jung, S. Lee, N. Park, All-optical 4-bit Gray code to binary coded decimal converter, Proc. SPIE 6890 (2008) 68900S1-10, 2008.

[4] A. Bhattacharyya, D.K. Gayen, T. Chattopadhyay, All-optical 4-bit binary to binary coded decimal converter with the help of semiconductor optical amplifier-assisted sagnac switch, Opt. Commun. 293 (2012) 31-42.

[5] A. G. Sasikala, S. Maragatharaj, S. Jayadevi, Effective binary to BCD converter using Quantum Dot Cellular Automata, Proc. IEEE on ICDCS (2014) 1-5.

[6] A. Bhattachryya, D. K. Gayen, T. Chattopadhyay, All-optical binary coded decimal to Binary converter with the help of terahertz asymmetric demultiplexer based switch, Proc. IEEE on CODEC (2012) 1-4.

[7] S. Kumar, G. Singh, A. Bisht, $4 \times 4$ signal router based on electrooptic effect of Mach-Zehnder interferometer for wavelength division multiplexing applications, Opt. Commun. 353 (2015) 17-26.

[8] S. Kumar, S.K. Raghuwanshi, A. Kumar, Implementation of full-adder and full-subtractor based on electro-optic effect in Mach-Zehnder interferometer, Opt. Commun.324 (2014) 93-107.

[9] S. K. Raghuwanshi, A. Kumar, N.K. Chen, Implementation of sequential logic circuits using the Mach-Zehnder interferometer structure based on electro-optic effect, Opt. Commun. 333(2014) 193208.

[10] S. Kumar, G. Singh, A. Bisht, A. Amphawan, Design of D flip-flop and T flip-flop using Mach-Zehnder interferometers for high-speed communication, Appl. Opt. 54 (2015) 6397-6405.

[11] S. Kumar, G. Singh, A. Bisht, K. Choudhary, D. Sharma, Implementation of wavelength selector based on electro-optic effect in Mach-Zehnder interferometers for high speed communications, Opt. Commun. 350,(2015) 108-118.

[12] S. K. Raghuwanshi, A. Kumar, S. Kumar, $1 \times 4$ signal router using three Mach-Zhender interferometers, Opt. Eng. 52(2013) 035002.

[13] S. Kumar, G. Singh, A. Bisht, A. Amphawan, Proposed new approach to design universal logic gate using Mach-Zehnder interferometers, Appl. Opt. 54(2015) 8479-8484.

[14] Santosh Kumar, Ashish Bisht, Gurdeep Singh, Kuldeep Choudhary, K. K. Raina, Angela Amphawan, "Implementation of 1-bit and 2-bit magnitude comparator using mach-zehnder interferometers," Optics Communications, 357 (2015) 127-147. 